\title{
Plasmid-based complementation of large deletions in Phaeodactylum tricornutum biosynthetic genes generated by Cas9 editing
}

Samuel S. Slattery ${ }^{1}$, Helen Wang ${ }^{1}$, Daniel J. Giguere ${ }^{1}$, Csanad Kocsis ${ }^{1}$, Bradley L. Urquhart ${ }^{2}$, Bogumil J. Karas ${ }^{1} \&$ David R. Edgell ${ }^{1 \bowtie}$

The model diatom Phaeodactylum tricornutum is an attractive candidate for synthetic biology applications. Development of auxotrophic strains of $P$. tricornutum would provide alternative selective markers to commonly used antibiotic resistance genes. Here, using CRISPR/Cas9, we show successful editing of genes in the uracil, histidine, and tryptophan biosynthetic pathways. Nanopore longread sequencing indicates that editing events are characterized by the occurrence of large deletions of up to $\sim 2.7 \mathrm{~kb}$ centered on the editing site. The uracil and histidine-requiring phenotypes can be complemented by plasmid-based copies of the intact genes after curing of the Cas9-editing plasmid. Growth of uracil auxotrophs on media supplemented with 5 -fluoroorotic acid and uracil results in loss of the complementing plasmid, providing a facile method for plasmid curing with potential applications in strain engineering and CRISPR editing. Metabolomic characterization of uracil auxotrophs revealed changes in cellular orotate concentrations consistent with partial or complete loss of orotate phosphoribosyltransferase activity. Our results expand the range of $P$. tricornutum auxotrophic strains and demonstrate that auxotrophic complementation markers provide a viable alternative to traditionally used antibiotic selection markers. Plasmid-based auxotrophic markers should expand the range of genome engineering applications and provide a means for biocontainment of engineered $P$. tricornutum strains.

Photoautotrophic microalgae and cyanobacteria are emerging as alternative platforms for synthetic biology applications ${ }^{1,2}$. One microalgae species of interest is the diploid marine diatom Phaeodactylum tricornutum. A variety of plasmid-based genetic tools have been developed for P. tricornutum that facilitate basic molecular manipulations and expression of complex synthetic pathways ${ }^{3-6}$. We, and others, have developed plasmid-based and DNA-free CRISPR (clustered regularly interspaced palindromic repeats) reagents for targeted chromosome editing in P. tricornutum and related diatoms using the Cas9 protein (CRISPR-associated protein 9) ${ }^{6-11}$. P. tricornutum is diploid, meaning that Cas9-edited cells must be carefully screened to determine if knockouts are monoallelic or biallelic and exhibit loss of heterozygosity. These plasmid-based tools and synthetic pathways are currently maintained by available antibiotic-based selections, including zeocin, phleomycin, nourseothricin, and blasticidin-S and their resistance genes, Sh ble, nat, and $b s r^{12-15}$. Antibiotic-based selections can be prohibitively expensive for maintaining large-scale cultures and are problematic for applications such as the biosynthesis of products intended for human consumption ${ }^{16-18}$.

A viable alternative to antibiotics is the use of auxotrophic selective markers which require a strain engineered to have a loss of function mutation in a key enzyme of an essential biosynthetic pathway. Examples of commonly used auxotrophic strains in industrial and academic labs include uracil, histidine, and tryptophan auxotrophs ${ }^{19-21}$. Two approaches have been taken to generate $P$. tricornutum auxotrophs. First, uracil-requiring mutants were generated by random mutagenesis that resulted in the identification of the bi-functional uridine monophosphate synthase (PtUMPS) gene predicted to catalyze the conversion of orotate into uridine monophosphate (UMP) $)^{22}$.

${ }^{1}$ Department of Biochemistry, Schulich School of Medicine and Dentistry, Western University, London, Canada. ${ }^{2}$ Department of Physiology and Pharmacology, Schulich School of Medicine and Dentistry, Western University, London, Canada. ${ }^{\boxplus}$ email: dedgell@uwo.ca 
Biolistic transformation and chromosomal integration of the PtUMPs gene rescued the uracil-requiring phenotype. Second, Cas9 was used to knockout the PtUMPS gene to create uracil auxotrophs and the PtAPT gene encoding a predicted adenine phosphoribosyl transferase to create adenine auxotrophs ${ }^{7}$. However, direct selection of these auxotrophs via transformation with the corresponding complementation marker has not been explored and the generation of additional auxotrophic strains would facilitate development of new plasmid-based complementation markers.

Here, we used a plasmid-based editing strategy to generate knockouts in the uracil, histidine, and tryptophan biosynthesis pathways of $P$. tricornutum and show for the first time that plasmid-based copies of the intact PtUMPS and PtPRA-PH/CH genes can complement the uracil- and histidine-requiring phenotypes, respectively. Individual auxotrophic strains are characterized by loss of heterozygosity at the edited alleles, and Nanopore sequencing of the edited population reveals large, heterogeneous deletions up to $\sim 2.7 \mathrm{~kb}$. The uracil and histidine auxotrophs and their respective complementation markers are a potential alternative to antibiotic-based selection of plasmids in P. tricornutum. Our results also suggest a simple methodology to cure plasmids from uracil auxotrophs to enable strain and genome engineering.

\section{Results}

Identification of Cas9 targets in biosynthetic pathway genes. We examined the KEGG predictions $^{23,24}$ based on the genome sequence of $P$. tricornutum to identify genes in the uracil and histidine biosynthetic pathways for Cas9 editing. We focused on these two pathways as uracil and histidine auxotrophy, and counter-selection strategies are commonly used in other model organisms. This approach identified the previously described bi-functional PtUMPS gene that is predicted to catalyze two steps in the uracil pathwayconversion of orotate to orotidine monophosphate (OMP), and conversion of OMP to uridine monophosphate (UMP) (Fig. 1A, Supplementary Fig. S1) ${ }^{22}$. Proteins that are orthologs of characterized enzymes involved in histidine biosynthesis were also identified (Fig. 2A, Supplementary Fig. S2). The PHATR_3140 gene, hereafter called PtPRA-PH/CH, encodes a predicted bifunctional protein that shares sequence similarity with the bacterial protein HisIE, and its plant counterpart HISN2 $2^{25,26}$. These proteins possess two functional domains that are homologous to the phosphoribosyl-ATP pyrophosphohydrolase (PRA-PH) and phosphoribosyl-AMP cyclohydrolase (PRA-CH) enzymes, respectively. PRA-PH and PRA-CH, alone or as a bifunctional protein, are predicted to catalyze two successive steps that occur early in the histidine biosynthesis pathway (Fig. 2A, Supplementary Fig. S2). The PtIGPS gene encoding imidazole glycerol phosphate synthase (a HIS3 homolog) was found to be a duplicated gene in the P. tricornutum genome assembly and thus not prioritized as a Cas 9 target.

We also identified the PtI3GPS-PRAI gene as a potential target as it encodes a predicted bi-functional enzyme that is a fusion of indole-3-glycerol-phosphate synthase (I3GPS) and phosphoribosylanthranilate isomerase (PRAI), and would catalyze two successive steps in the tryptophan biosynthesis pathway (Supplementary Fig. S3).

To confirm the genomic target sites, we PCR-amplified and sequenced the PtUMPS and PtPRA-PH/CH genes of the P. tricornutum CCAP 1055/1 strain used in our laboratory. Two distinct alleles for both the PtUMPS and PtPRA-PH/CH genes were identified. Seven single-nucleotide polymorphisms (SNPs) in the PtUMPS alleles result in amino acid substitutions that differentiate the two alleles from each other and from the published $P$. tricornutum genome (Supplementary Table S1). All substitutions are located in non-conserved regions of the PtUMPS protein (Supplementary Fig. S4). Similarly, an A to G mutation at base position 1205 in allele 2 of the PtPRA-PH/CH gene was identified (Supplementary Table S1). This transversion converts a highly conserved glutamate to a glycine in the catalytic site of the PRA-PH domain. The impact of these substitutions on PtUMPS and PtPRA-PH/CH function is unknown.

Cas9 and TevCas9 editing of auxotrophic genes is characterized by loss of heterozygosity. To generate knockouts in uracil and histidine biosynthetic genes, we designed and individually cloned Cas9 and TevCas9 single guide RNAs (sgRNAs) against different sites in the PtUMPS, PtPRA-PH/CH, and PtI3GPS-PRAI genes (Table 1, Supplementary Fig. S5-S7). The TevCas9 nuclease is a dual nuclease that generates a 33-38 base pair deletion between the I-TevI (Tev) and Cas 9 cut sites ${ }^{27}$. The targeting requirements for a TevCas 9 nuclease are an I-TevI $5^{\prime}$-CNNNG-3' cleavage motif positioned $\sim 15-18$ base pairs upstream of the $5^{\prime}$ end of the sgRNA binding site. The Cas 9 or TevCas9 editing plasmids were moved into P. tricornutum by bacterial conjugation and exconjugants selected on zeocin-containing media.

We first assessed editing by screening $P$. tricornutum exconjugants by T7 endonuclease I (T7EI) mismatch cleavage assays on PCR products amplified from each target gene (Figs. 1B, 2B, Table 1). This assay identified 6 sgRNAs with detectable editing rates based on screening of exconjugants. Colonies that showed editing were diluted, plated to obtain subclones, and subsequently screened for the corresponding auxotrophic phenotype on solid media with and without auxotrophic supplement (uracil or histidine) (Figs. 1C, 2C). To cure the Cas9editing plasmids, knockout strains were grown without zeocin selection for 1 week, and dilutions were plated to obtain single colonies. Colonies were streaked onto L1 plates with and without zeocin to screen for plasmid loss. A representative image demonstrating zeocin sensitivity due to loss of the Cas9-editing plasmid is shown in Fig. 1D. For knockout of the PtUMPS gene, we further characterized 3 subclones with a uracil-requiring phenotype to determine if the knockouts were monoallelic or biallelic. Because the two PtUMPS alleles of P. tricornutum possessed SNPs relative to each other, we were able to map allele-specific editing events (Fig. 1E,F). Two of the strains, $\triangle$ UMPS1 and $\triangle$ UMPS2, were biallelic and exhibited loss of heterozygosity with one allele possessing a small deletion $(<20 \mathrm{bps})$ and the other allele possessing a large deletion $(>610 \mathrm{bp})$. The third characterized subclone, $\triangle \mathrm{UMPS} 3$, was monoallelic and possessed a homozygous 1-bp insertion. For the PtPRA-PH/CH knockouts that generated a histidine-requiring phenotype (Fig. 2B,C), targeted sequencing of one subclone revealed a biallelic genotype with an 11-bp deletion in one allele and a 6-bp deletion in the second allele (Fig. 2D,E). 
A

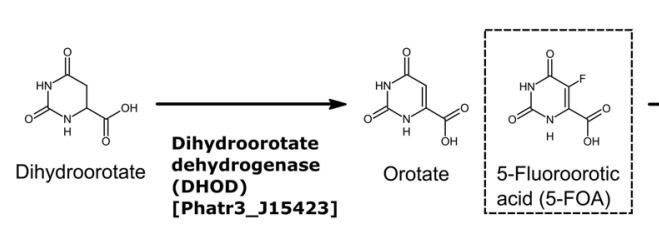

B

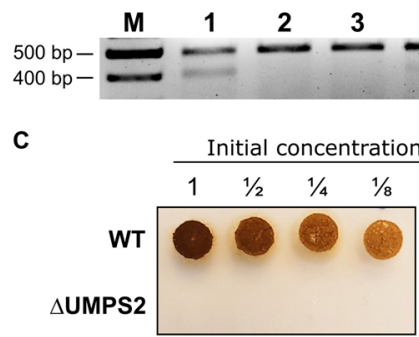

L1
$4 \quad 5$

5

6 WT - Substrate
-T7 product

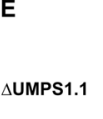

$\triangle$ UMPS2.1

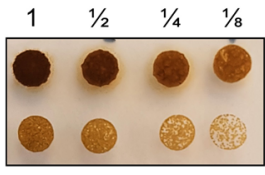

L1 + Uracil

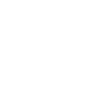

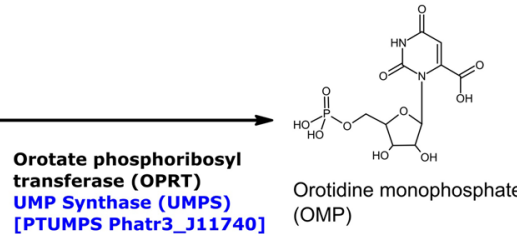

MWMWMWMWM

TGGTAAATTGGGAAAACGAG G1636' 'G1655 17 bp del WWMMMWWMM GTGGTAAATTAAGGAAAACG T1635' A1653

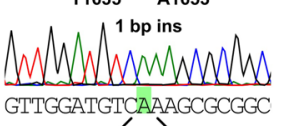
C309' 'A310

AUMPS3.1/2 GTTGGATGTCAAAG

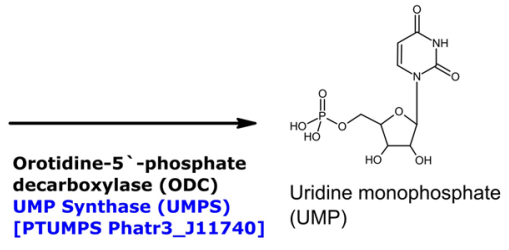

[PTUMPS Phatr3_J11740] (UMP)

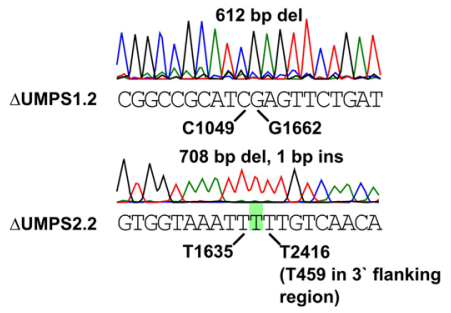

region)

D

Initial concentration $\left(\times 10^{4}\right.$ cells plaque $^{-1}$ )

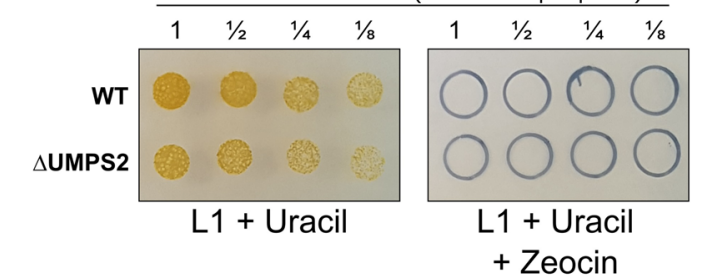

F

SgRNA.UMPS.311

UMPS

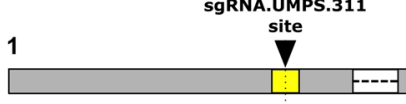

sgRNA.UMPS.1646

$\triangle$ UMPS1.1 18 bp del

$\triangle$ UMPS1.2 612 bp del

$\triangle$ UMPS2.1 17 bp del

$\triangle$ UMPS2.2 708 bp de

$1 \mathrm{bp}$ ins

$\triangle$ UMPS3.1/2 1 bp ins

Figure 1. CRISPR-generated knockouts in the predicted P. tricornutum uracil biosynthesis pathway. (A) A portion of the predicted $P$. tricornutum biosynthesis pathway for conversion of carbonic acid to uracil and uridine triphosphate, with the PtUMPS enzyme highlighted in blue. The competitive inhibitor, 5-fluoroorotic acid (5-FOA), is shown in a dashed box at the position where it enters the pathway. Abbreviated names for molecules and enzymes are indicated in parentheses, and the predicted corresponding $P$. tricornutum gene names are indicated in square brackets. (B) Example image of T7EI editing assay to screen exconjugants for potential editing events in the PtUMPS gene. Substrate indicates PtUMPS gene fragments amplified by the PCR, while T7 product indicates exconjugants with evidence of Cas9 editing. WT, wild-type $P$. tricornutum genomic DNA used in the T7EI editing assay. M, 100 bp ladder with sizes indicated in basepairs (bp). This image was cropped from a larger image (Supplementary Fig. S10). (C) Example of phenotypic screening of one PtUMPS knockout strain ( $\triangle$ UMPS2) plated on L1 alone or L1 supplemented with uracil at the indicated dilution of initial concentration. (D) Example of screening for loss of the zeocin-resistant Cas9 editing plasmid in a $\Delta$ UMPS2 knockout strain by plating on L1 supplemented with uracil or L1 supplemented with uracil and zeocin. (E) Sanger sequencing traces of characterized PtUMPS knockouts with the position (below trace) and type of insertion or deletion (above trace) indicated for each allele of the three strains. (F) Graphical map of the position and extent of indels for each of the three PtUMPS knockouts relative to the wild-type UMPS gene (shown at top). Red rectangles indicate nucleotide deletions, green triangles indicate nucleotide insertions, the yellow and blue rectangles on the WT gene indicate the position of the PtUMPS active sites (orotate phosphoribosyl transferase and orotidine-5'-phosphate decarboxylase), and the white rectangles with dashed lines represent introns. 
A<smiles>CC1C(OCC(C)(C)C)OC(CC(C)(C)C)C1OC(C)(C)C</smiles>

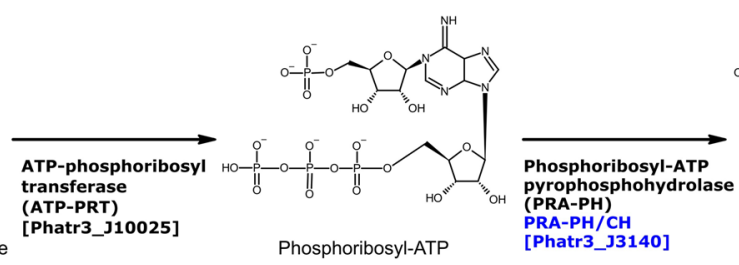

Phosphoribosylpyrophosphate (ATP-PRT)

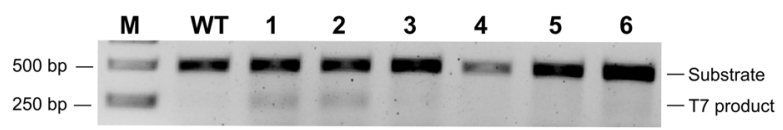

C

Initial concentration ( $\times 10^{4}$ cells plaque ${ }^{-1}$ )

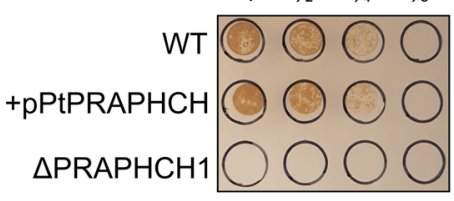

L1

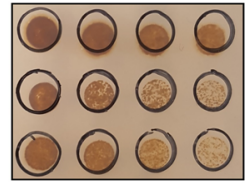

L1 + Histidine
D

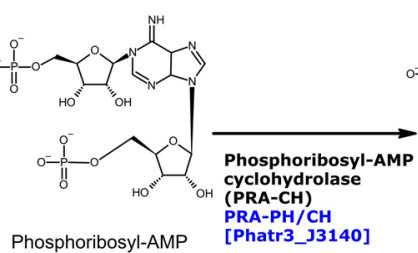

$11 \mathrm{bp}$ del

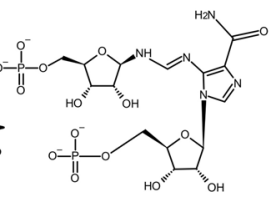

Phosphoribosylformimino -AICAR-phosphate

E

PRA-PH/CH

$\triangle$ PRAPHCH1.1 11 bp del

$\triangle$ PRAPHCH1.2 6 bp del

Figure 2. CRISPR-generated knockouts in the predicted P. tricornutum histidine biosynthesis pathway. (A) A portion of the predicted biosynthesis pathway for conversion of ribose-5-phosphate to L-histidine, with the bi-functional PtPRA-PH/CH enzyme highlighted in blue. Abbreviated names for each enzyme are indicated in parentheses, and the predicted corresponding $P$. tricornutum gene names are indicated in square brackets. (B) Example image of T7EI editing assay to screen exconjugants for potential editing events in the PtPRA-PH/ $\mathrm{CH}$ gene. Substrate indicates PtPRA-PH/CH gene fragments amplified by the PCR, while T7 product indicates exconjugants with evidence of Cas 9 editing. WT, wild-type $P$. tricornutum genomic DNA used in the T7EI editing assay. M, $1 \mathrm{~kb}$ ladder with sizes indicated in basepairs (bp). This image was cropped from a larger image (Supplementary Fig. S11). (C) Example of phenotypic screening of one PtPRA-PH/CH knockout strain $(\Delta$ PtPRAPHCH1) transformed with or without the complementing PRA-PH/CH plasmid (pPtPRAPHCH) on L1 solid media alone or L1 supplemented with histidine at the indicated dilution of initial concentration. WT, wild-type $P$. tricornutum strain. (D) Sanger sequencing traces of characterized PtPRA-PH/CH knockouts with the position (below trace) and type of insertion or deletion (above trace) indicated for each allele. (E) Graphical map of the position and extent of indels for PtPRA-PH/CH knockout relative to the wild-type PtPRA-PH/CH gene (shown at top). Red rectangles indicate nucleotide deletions, while the yellow and blue rectangles on the WT gene indicated the position of the PRA-PH and PRA-CH active sites.

\begin{tabular}{|c|c|c|c|c|}
\hline Target & Platform & Guide RNA & $\begin{array}{l}\text { Exconjugants edited / total screened } \\
\text { (T7E1) }\end{array}$ & $\begin{array}{l}\text { Number of subclones with } \\
\text { auxotroph phenotype / screened }\end{array}$ \\
\hline \multirow{7}{*}{ UMPS } & \multirow{4}{*}{ Cas9 } & sgRNA.UMPS.1944 & $0 / 10$ & N/A \\
\hline & & sgRNA.UMPS.1646 & $0 / 10$ & N/A \\
\hline & & sgRNA.UMPS.157 & $0 / 10$ & N/A \\
\hline & & sgRNA.UMPS.311 & $4 / 10$ & $1 / 35$ \\
\hline & \multirow{3}{*}{ TevCas9 } & sgRNA.UMPS.1944 & $0 / 10$ & N/A \\
\hline & & sgRNA.UMPS.1646 & $4 / 10$ & $2 / 35$ \\
\hline & & sgRNA.UMPS.157 & $0 / 10$ & N/A \\
\hline \multirow{5}{*}{ PRA-PH/CH } & \multirow{3}{*}{ Cas9 } & sgRNA.PRAPHCH.929 & $2 / 6$ & $1 / 28$ \\
\hline & & sgRNA.PRAPHCH.120 & $0 / 6$ & N/A \\
\hline & & sgRNA.PRAPHCH.1000 & $1 / 6$ & $0 / 28$ \\
\hline & \multirow{2}{*}{ TevCas9 } & sgRNA.PRAPHCH.929 & $3 / 6$ & $0 / 28$ \\
\hline & & sgRNA.PRAPHCH.120 & $0 / 6$ & N/A \\
\hline \multirow{2}{*}{ I3GPS-PRAI } & Cas9 & sgRNA.IGPSPRAI.244 & $0 / 10$ & N/A \\
\hline & \begin{tabular}{|l|} 
TevCas9 \\
\end{tabular} & sgRNA.IGPSPRAI.244 & $3 / 10$ & $1 / 35$ \\
\hline
\end{tabular}

Table 1. Summary of sgRNAs used for Cas9 and TevCas9 editing. sgRNAs are named by the first nucleotide of the sgRNA binding site in the coding region of the target gene. 
The types of deletions observed in the uracil- and histidine-auxotrophs are consistent with heterogeneous editing events resulting in loss of heterozygosity ${ }^{28-30}$. To extend these observations, we used Nanopore sequencing to better assess the spectrum of large deletions that are often overlooked in Cas9-editing studies. In addition to the two sgRNAs that showed robust editing on the PtUMPS gene, we examined deletion events in exconjugants with sgRNAs targeted to the PtUREASE gene ${ }^{6}$ and the PtI3GPS-PRAI gene. For each experiment, $\sim 1,000$ exconjugants were pooled and $\mathrm{a} \sim 6 \mathrm{~kb}$ PCR product generated for each of the target genes with the predicted Cas 9 or TevCas 9 target sites in the middle of the amplicon. We focused our attention on deletions $>50$ bp as these deletions are typically under-reported in targeted amplicon sequencing. We noted a drop in Nanopore read coverage centered around the predicted sgRNA target sites for products amplified from Cas 9 and TevCas9 editing experiments (black dots) as compared to read coverage for control experiments (orange dots), consistent with editing at those sites (Fig. 3, left panels). Mapping the deletion start and end points revealed that most deletions were centered on the Cas9 or TevCas9 target site (Fig. 3, right panels), with deletions extending up to $2700 \mathrm{bp}$ (Fig. 3, centre panel). The mean deletion length for editing events examined by Nanopore sequencing and $>50$ bp was $1735 \pm 719$ bp for Cas 9 and $2006 \pm 633$ bp for TevCas9.

Collectively, this data shows that Cas 9 or TevCas 9 editing of biosynthetic genes can readily generate $P$. tricornutum auxotrophs that can be identified by phenotypic or genetic screens. Moreover, our data agree with a growing body of evidence revealing that Cas9 editing (and TevCas9 editing here) generates large deletions that would typically be missed unless screening strategies are explicitly designed to look for loss of heterozygosity.

Phenotypic and metabolomic characterization of the PtUMPS knockouts. Two uracil-requiring auxotrophs ( $\triangle$ UMPS1 and $\triangle$ UMPS2) were selected for further characterization by first spot plating onto L1 media with and without uracil and 5-FOA (Fig. 4A). The PtUMPS knockout strains were only able to survive in the presence of uracil supplementation. Additionally, the knockouts survived on 5-FOA concentrations that fully inhibited the growth of wild-type P. tricornutum (Fig. 4A). This is consistent with phenotypes previously observed for P. tricornutum UMPS knockouts ${ }^{7,22}$. There was a slight growth advantage of $\triangle$ UMPS1 over $\triangle$ UMPS2 on media supplemented with both 5-FOA and uracil, but not on media containing uracil alone. To compare if the observed phenotypes were consistent across solid and liquid media, we monitored the growth of these strains over 10 days in liquid media (Fig. 4B) and found that the growth rates were consistent with those observed on solid media, with one notable difference (Supplementary Figs. S8, S9, Supplementary Table S3). The growth advantage of $\triangle \mathrm{UMPS} 1$ over $\triangle \mathrm{UMPS} 2$ observed on solid media supplemented with both 5-FOA and uracil was not replicated in liquid media as the generation times for $\triangle$ UMPS1 and $\triangle$ UMPS2 were very similar $(\sim 24$ and $\sim 22$ h, respectively $)$.

To investigate the impact of PtUMPS knockouts on uracil metabolism, we performed targeted metabolomics on the UMPS substrate orotate using LC-MS in wild-type and knockouts strains (Fig. 4C). We focused on characterizing the orotate intermediate in the uracil pathway (Figs. 2A, 4C) predicting that there should be an increase of orotate in knockout strains relative to wild type. We were unable to detect orotate in the $\triangle$ UMPS1 strain in the absence of uracil supplementation (-uracil), or in the $\triangle$ UMPS2 strain in either the -uracil or +uracil condition. A sixfold increase of cellular orotate levels was observed in the wild-type strain when L1 media was supplemented with uracil (+uracil) as compared to minimal L1 media (-uracil) (Fig. 4C). Interestingly, when the $\triangle$ UMPS1 strain was grown with uracil supplementation we detected orotate at levels similar to those observed in the wild-type strain grown with uracil. This result suggests that allele 1 in the $\triangle$ UMPS1 knockout strain (with an 18-bp in-frame deletion) retains UMPS activity that behaves similarly to the wild-type strain. In contrast, the $\triangle$ UMPS2 strain has two out-of-frame deletions that likely abolish ODC and OPRT activity. We speculate that undetectable levels of orotate in the $\triangle$ UMPS2 strain may be because it is diverted to another biosynthetic pathway.

Plasmid complementation of the uracil and histidine auxotrophs. Plasmid-based complementation of P. tricornutum auxotrophs would validate that the Cas9-editing event was the cause of the auxotrophic phenotype, as well as providing alternatives to antibiotic-based selection methods to maintain episomal vectors. We first examined complementation of the uracil-requiring phenotype by cloning both gDNA and cDNA versions of each PtUMPS allele with the native promoter and terminator into the nourseothricin-resistant pPtGE31 expression plasmid ${ }^{6}$. These plasmids were designated pPtUMPSA1, pPtUMPSA2, pPtUMPScA1, and pPtUMPScA2 (Supplementary Table S3) and moved into the $\triangle$ UMPS1 and $\triangle$ UMPS2 strains via conjugation. Exconjugants were spot-plated onto solid L1 media with and without uracil and 5-FOA supplementation (Fig. 4A). All complemented strains grew on minimal L1 media, while the uncomplemented knockouts did not, confirming expression of the UMPS gene from the pPtGE31 plasmid. No strain grew on 5-FOA alone. Unexpectedly, some of the complemented strains survived on plates supplemented with both 5-FOA and uracil. For example, when $\triangle$ UMPS2 was transformed with either of the allele 1 complementation plasmids (pPtUMPSA1 and pPtUMPScA1), clear resistance to 5-FOA in the presence of uracil was observed. The phenotypes observed on solid media were consistent with those observed when the strains were grown in liquid media with similar media supplementation (Supplementary Figs. S8, S9).

The growth phenotype of the $\triangle \mathrm{UMPS} 1$ and $\triangle \mathrm{UMPS} 2$ strains in media supplemented with uracil and 5-FOA could be explained by counter-selection against the plasmid carrying an intact PtUMPS gene that would metabolize 5-FOA to a toxic intermediate. We thus tested for plasmid loss in the complemented strains by plating the $\Delta$ UMPS1 and $\triangle$ UMPS2 strains carrying different expression plasmids on solid L1 with and without nourseothricin after 14 days of growth. As shown in Fig. 4D, plasmid retention, as measured by the ratio of colonies on L1 plus nourseothricin versus L1 plates, was severely reduced in all strains, ranging from $\sim 1$ to $\sim 33 \%$. This observation could explain why colonies readily appeared on L1 media supplemented with 5-FOA and uracil and suggest that 
A TRP1: Cas9 sgRNA.IGPSPRAI.244
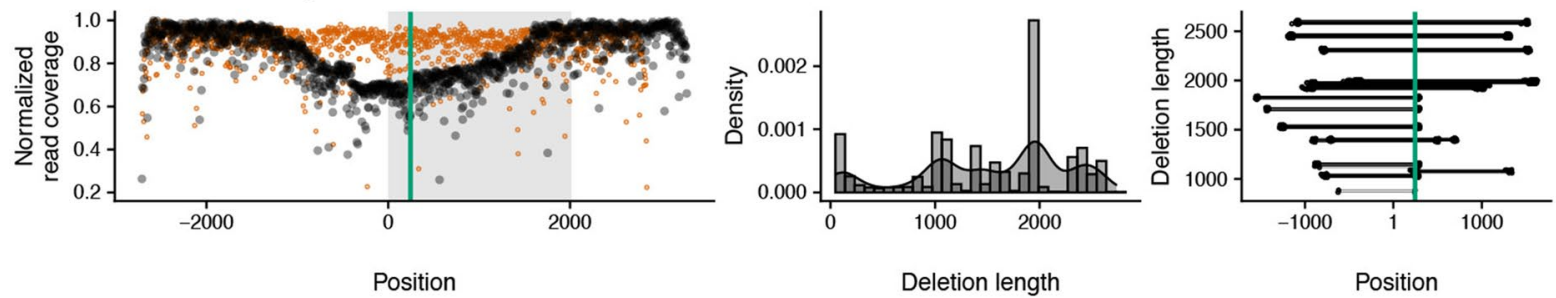

B TRP1: TevCas9 sgRNA.IGPSPRAI.244
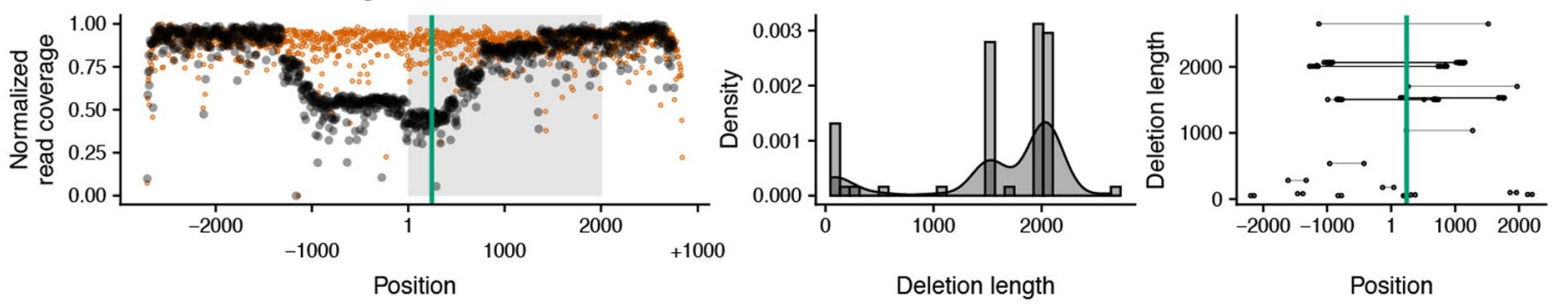

C UMPS: Cas9 sgRNA.UMPS.1944
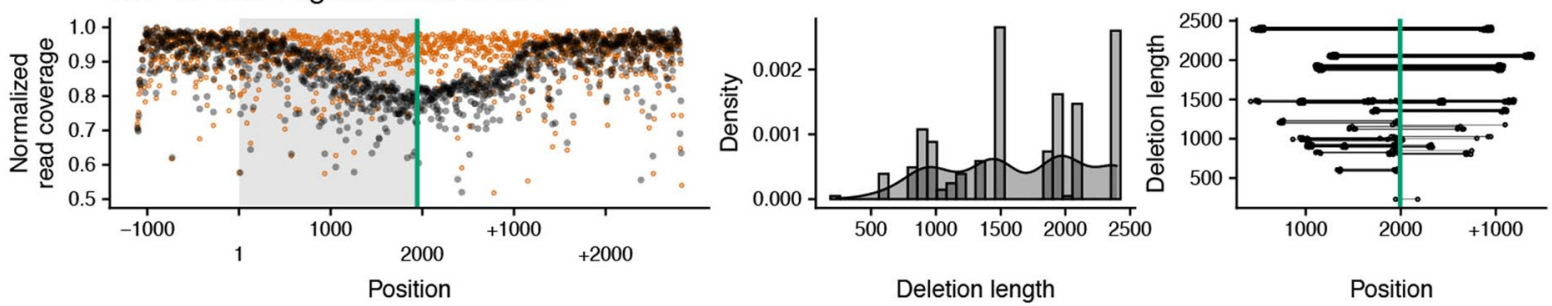

D UMPS: TevCas9 sgRNA.UMPS.1944
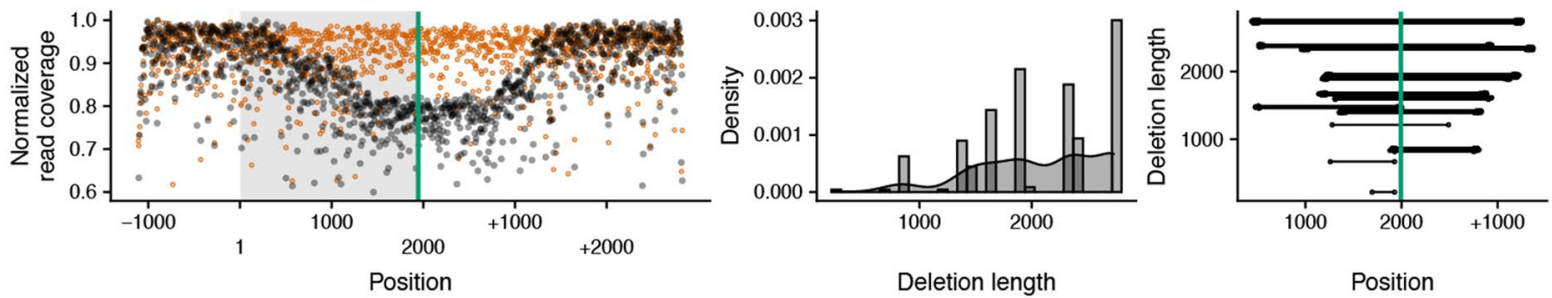

E UMPS: Cas9 sgRNA.UMPS.311
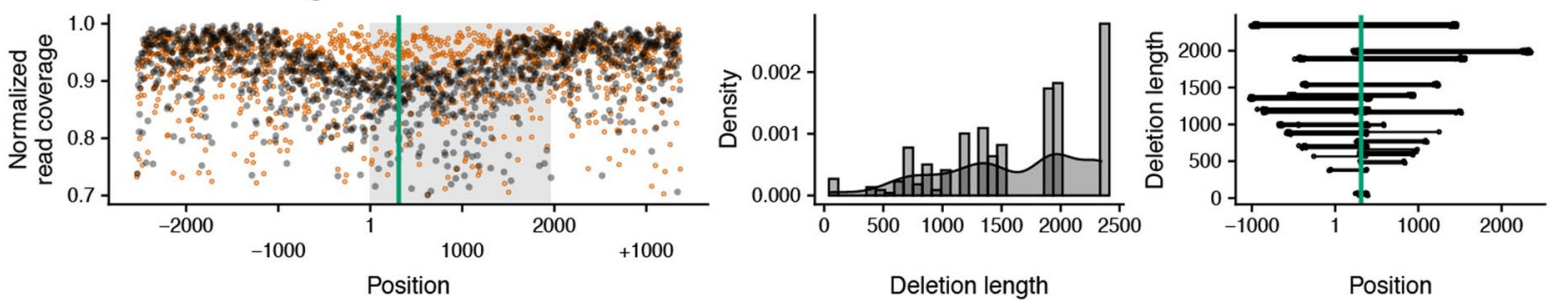

F

UREASE: Cas9 sgRNA.UREASE.1187
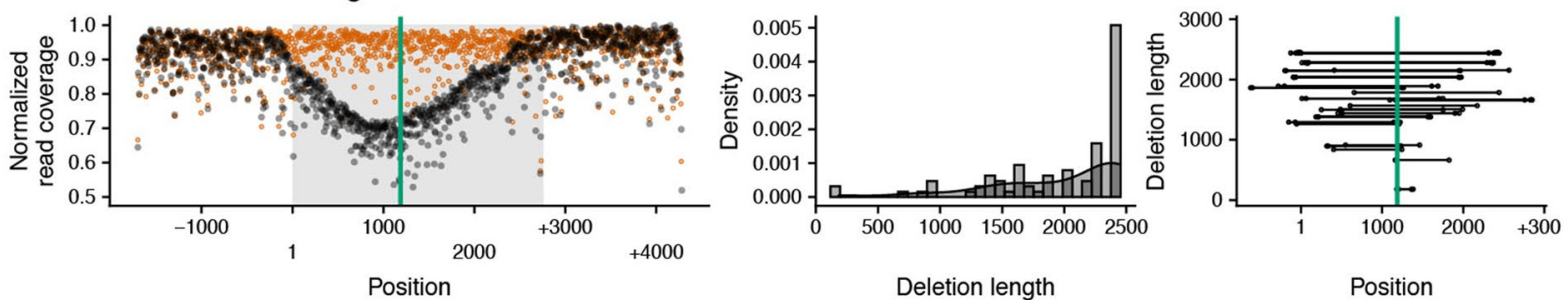
4Figure 3. Large deletions in edited $P$. tricornutum metabolic genes captured by Nanopore amplicon sequencing. For each (A-E), the name of the target gene as well as the editing enzyme are indicated. The leftmost plot shows normalized read coverage averaged over a 5-bp window for the edited sample (black dots) and the wild-type sample (orange dots) relative to the position in PCR amplicon. Numbering on the $\mathrm{x}$-axis is relative to the ATG start codon for each gene, with sequence upstream indicated by a minus (-) symbol and sequence downstream indicated by a plus (+) symbol. The green vertical line indicates the Cas 9 or TevCas 9 cleavage site, while the shaded rectangle indicates the ORF. The middle plot is a density plot of deletions $>50-\mathrm{bp}$. The rightmost plot shows the length and position of deletions $>50$-bp relative to their position in the PCR amplicon, with numbering of the $\mathrm{x}$-axis as in the leftmost panel. Each horizontal line indicates a mapped deletion event. Deletions are ordered from longest to smallest. The green line indicates the Cas 9 or TevCas 9 cleavage site.

curing of plasmids carrying the PtUMPS gene from PtUMPS knockout strains is a simple matter of growth on the appropriate media.

Similarly, we were able to complement the histidine-requiring phenotype by cloning a wild-type copy of the PtPRA-PH/CH gene into an expression vector, and transforming the plasmid into the $\triangle$ PRAPHCH1 strain by conjugation. The $\triangle \mathrm{PRAPHCH} 1$ strain with the complementing plasmid grew on both solid L1 media with and without histidine supplementation, whereas the $\triangle \mathrm{PRAPHCH} 1$ strain without the complementing plasmid only grew on L1 media with histidine supplementation (Fig. 2C).

\section{Discussion}

The available tools for genetic manipulation of $P$. tricornutum and other diatoms have grown substantially in recent years, including the adaptation of TALEN and Cas9 genome-editing nucleases for targeted knockouts as well as plasmid-based and DNA-free methods to deliver the nucleases to cells $s^{6-9,11,31,32}$. Applications of genomeediting nucleases in P. tricornutum at this point have mostly been to generate gene knockouts, with a few examples of reporter construct knockins. Generation of gene knockouts by Cas 9 or other editing enzymes relies on non-homologous end-joining repair pathways ${ }^{33}$, homologs of which are predicted to occur in the P. tricornutum genome ${ }^{23,24}$. A recent study used antisense RNA to knockdown a predicted DNA ligase IV homolog $($ ligIV) in $P$. tricornutum resulting in an increased rate of homologous recombination of a reporter construct ${ }^{34}$. What is not yet known for P. tricornutum is the balance between NHEJ and homology directed repair (HDR) pathways that process endonuclease-introduced double-strand breaks (see for example ${ }^{35}$ ). Examination of Cas9 or TALENedited sites in P. tricornutum revealed small nucleotide insertions or deletions localized near the editing site that are consistent with NHEJ repair events. It is becoming increasingly apparent that repair of Cas9-edited sites result in heterogenous alleles often characterized by both small and large deletions (for example, Fig. 1D) ${ }^{6,8,36}$. Repair events leading to large indels are often missed by experimental strategies that examine repair outcomes localized around the editing site. In contrast, large deletions visible by long-read sequencing methodologies and our Nanopore data indicate that Cas 9 and TevCas 9 editing events result in deletions up to $\sim 2.7 \mathrm{~kb}$ in length. Cas 9 editing with a single sgRNA in P. tricornutum could achieve the same goal as the paired Cas 9 nickase strategy to specifically introduce large deletions $s^{37}$, and may be complementary with recently developed methods to multiplex sgRNAs on Cas9-editing plasmids for P. tricornutum ${ }^{10}$. Regardless, a better understanding of DNA repair pathways that operate on Cas9-introduced double-strand breaks will better inform strategies to bias repair events depending on the experimental goal.

The creation of auxotrophic strains of $P$. tricornutum with plasmid based rather than chromosomally integrated complementation markers is critical for a number of reasons. Auxotrophic strains expand the available selection schemes beyond traditional antibiotic markers and provide a facile method for strain cataloging and validation. Antibiotic-free selection is also an advantage when P. tricornutum is used for production of human therapeutics. In the case of uracil auxotrophs, complementing plasmids can be cured (or counter selected) by simple inclusion of 5-FOA and uracil in the growth media. We have previously shown that plasmids are lost from $P$. tricornutum by passaging cultures over multiple days in the absence of antibiotic selection required for maintenance of the plasmid ${ }^{38}$. However, the counter selection method by 5 -FOA and uracil supplementation is more rapid and requires screening significantly fewer colonies to confirm plasmid loss. The ability to rapidly cure plasmids will be of tremendous value to prevent prolonged expression of Cas 9 and possible toxicity issues during strain engineering, to cure incompatible plasmids, or to cure reporter or expression plasmids under distinct growth conditions. We also envision that rapid curing of plasmids would allow recycling of a limited number of selection markers for serial transformations needed for strain construction or genomic engineering.

\section{Methods}

Microbial strains and growth conditions. Saccharomyces cerevisiae VL6-48 (ATCC MYA-3666: MAT $\alpha$ his 3- 200 trp 1- $\delta 1$ ura3-52 lys2 ade2-1 met $14^{\circ}$ ) was grown in rich medium (YPD) or complete minimal medium lacking histidine (Teknova) supplemented with $60 \mathrm{mg} \mathrm{L}^{-1}$ adenine sulfate. Complete minimal media used for spheroplast transformation contained $1 \mathrm{M}$ sorbitol. Escherichia coli (Epi300, Epicenter) was grown in Luria Broth (LB) supplemented with appropriate antibiotics (chloramphenicol $25 \mathrm{mg} \mathrm{L}^{-1}$ or kanamycin $50 \mathrm{mg} \mathrm{L}^{-1}$ or ampicillin $50 \mathrm{mg} \mathrm{L}^{-1}$ or gentamicin $20 \mathrm{mg} \mathrm{L}^{-1}$ ). Phaeodactylum tricornutum (Culture Collection of Algae and Protozoa CCAP 1055/1) was grown in L1 medium without silica, with or without uracil $\left(50 \mathrm{mg} \mathrm{L}^{-1}\right)$ or histidine $\left(200 \mathrm{mg} \mathrm{L}^{-1}\right)$ or 5-FOA $\left(100 \mathrm{mg} \mathrm{L}^{-1}\right)$, supplemented with appropriate antibiotics zeocin $\left(50 \mathrm{mg} \mathrm{L}^{-1}\right)$ or nourseothricin $\left(100 \mathrm{mg} \mathrm{L}^{-1}\right)$, at $18^{\circ} \mathrm{C}$ under cool white fluorescent lights $\left(75 \mu \mathrm{E} \mathrm{m}^{-2} \mathrm{~s}^{-1}\right)$ and a photoperiod of $16 \mathrm{~h}$ light:8 h dark. L1 media supplemented with nourseothricin contained half the normal amount of aquil salts. P. tricornutum auxotroph genotypes are as follows. Mutations in PtUMPS are described in reference to the 
Figure 4. Phenotypic and metabolomic characterization of PtUMPS knockouts. (A) Spot plating assays of wild type (WT), $\triangle \mathrm{UMPS} 1$ and $\triangle \mathrm{UMPS} 2$ strains on L1 solid media alone, L1 supplemented with uracil, L1 supplemented with 5-FOA, or L1 supplemented with both uracil and 5-FOA. Indicated dilutions are relative to the initial concentration. (B) Liquid growth curves of wild type (WT), $\triangle \mathrm{UMPS} 1$ and $\triangle \mathrm{UMPS} 2$ strains in L1 liquid media alone, or supplemented with uracil or 5-FOA or both. Data points are the mean of three independent replicates, with error bars representing the standard error of the mean. (C) Orotate concentrations were measured by LC-MS from cultures grown with and without uracil supplementation. Bars represent mean values and error bars represent standard deviation for three biological replicates. Individual data points are represented as colored dots. Statistical confidence level was calculated by one-sided t test. $\mathrm{p}<0.001$ is indicated by an asterisk. (D) Bar graph showing percent plasmid retention in the $\triangle$ UMPS1 and $\triangle$ UMPS2 strains harbouring various PtUMPS constructs after 14 days of outgrowth. Bars represent the mean ratio of colonies on selective L1 + nourseothricin versus non-selective L1 plates from three independent replicates, with error bars representing the standard error of the mean.

chromosome 6 sequence (GenBank: CM000609.1), and mutations for PtPRA-PH/CH are in reference to the chromosome 3 sequence (GenBank: CP001142.1). Mutations described for each gene are listed for allele 1 followed by allele 2, and numbered beginning from the first nucleotide of the start codon for simplicity. Genotypes of auxotroph strains generated in this study are listed in Supplementary Table S4.

Transfer of DNA to $P$. tricornutum via conjugation from $\boldsymbol{E}$. coli. Conjugations were performed as previously described ${ }^{5,6}$. Briefly, liquid cultures $(250 \mu \mathrm{L})$ of $P$. tricornutum were adjusted to a density of $1.0 \times 10^{8}$ cells $\mathrm{mL}^{-1}$ using counts from a hemocytometer, plated on $1 / 2 \times \mathrm{L} 11 \%$ agar plates and grown for four days. L1 media $(1.5 \mathrm{~mL})$ was added to the plate and cells were scraped and the concentration was adjusted to $5.0 \times 10^{8}$ cells $\mathrm{mL}^{-1}$. E. coli cultures $(50 \mathrm{~mL})$ were grown at $37^{\circ} \mathrm{C}$ to $\mathrm{A} 600$ of $0.8-1.0$, centrifuged for $10 \mathrm{~min}$ at $3,000 \times g$ and resuspended in $500 \mu \mathrm{L}$ of SOC media. Conjugation was initiated by mixing $200 \mu \mathrm{L}$ of $P$. tricornutum and 200 $\mu \mathrm{L}$ of $E$. coli cells. The cell mixture was plated on $1 / 2 \times \mathrm{L} 15 \% \mathrm{LB} 1 \%$ agar plates, incubated for $90 \mathrm{~min}$ at $30^{\circ} \mathrm{C}$ in the dark, and then moved to $18^{\circ} \mathrm{C}$ in the light and grown for 2 days. After 2 days, $\mathrm{L} 1$ media $(1.5 \mathrm{~mL})$ was added to the plates, the cells scraped, and $300 \mu \mathrm{L}(20 \%)$ plated on $1 / 2 \times \mathrm{L} 11 \%$ agar plates supplemented with zeocin 50 $\mathrm{mg} \mathrm{L}^{-1}$ or nourseothricin $200 \mathrm{mg} \mathrm{L}^{-1}$. Colonies appeared after $7-14$ days incubation at $18^{\circ} \mathrm{C}$ with light.

Plasmid design and construction. All plasmids (Supplementary Table S5) were constructed using a modified yeast assembly protocol ${ }^{39,40}$. Plasmids pPtUMPSA1 and pPtUMPSA2 were made from pPtGE $31^{6}$ by replacing the URA3 element with a PCR fragment consisting of PtUMPS allele 1 or 2 with $\sim 1 \mathrm{~kb}$ up- and downstream of the PtUMPS ORF amplified from P. tricornutum genomic DNA (oligonucleotides are listed in Supplementary Table S6). Plasmids pPtUMPScA1 and pPtUMPScA2 were made from pPtUMPSA1 and pPtUMPSA2 by replacing the PtUMPS ORF with a PCR fragment consisting of PtUMPS allele 1 or 2 amplified from $P$. tricornutum cDNA. Plasmid pPtUMPS40S was made from pPtGE31 by replacing the URA3 element with a cassette consisting of PCR fragments of the 40SRPS8 promoter and terminator ${ }^{6}$ flanking a PCR fragment of the PtUMPS allele 1 ORF amplified from $P$. tricornutum genomic DNA. Plasmid pPtPRAPHCH was made from pPtGE31 ${ }^{6}$ by replacing the URA3 element with a PCR fragment consisting of PtPRA-PH/CH with $\sim 1 \mathrm{~kb}$ up- and downstream of the PtPRA-PH/CH ORF, amplified from P. tricornutum genomic DNA. Using Golden Gate assembly, sgRNAs targeting different regions of the PtUMPS and PtPRA-PH/CH genes were cloned into the BsaI sites positioned between the $P$. tricornutum U6 promoter and terminator in pPtGE34 and pPtGE35. Plasmid constructs were confirmed by Sanger sequencing at the London Regional Genomics Facility.

Generation of PtUMPS and PtPRA-PH/CH knockouts using Cas9 and TevCas9. Plasmids pPtGE34 or pPtGE35, containing no guide RNA or sgRNA.UMPS.1944, sgRNA.UMPS.1646, sgRNA. UMPS.157, sgRNA.UMPS.311 for the PtUMPS gene, or sgRNA.PRAPHCH.929 or sgRNA.PRAPHCH.120 for the PtPRA-PH/CH gene, were conjugated from E. coli to $P$. tricornutum and exconjugants were selected on zeocin-containing media, supplemented with uracil or histidine as appropriate ${ }^{41}$. Ten colonies from each conjugation were resuspended in TE buffer and flash frozen at $-80^{\circ} \mathrm{C}$ followed by heating at $95^{\circ} \mathrm{C}$ to lyse cells and extract genomic DNA. The genomic target site of each sgRNA in P. tricornutum was amplified by PCR and the products were analyzed by T7EI assay as follows; PCR products were denatured at $95^{\circ} \mathrm{C}$ for 5 min, slowly cooled to $50^{\circ} \mathrm{C}$, and flash frozen at $-20^{\circ} \mathrm{C}$ for $2 \mathrm{~min}$. PCR products $(250 \mathrm{ng}$ ) were incubated with $2 \mathrm{U}$ of T7EI (NEB) in $1 \times$ NEBuffer 2 for $15 \mathrm{~min}$ at $37^{\circ} \mathrm{C}$ and analyzed by agarose gel electrophoresis. Colonies that showed editing by T7EI assay were grown in liquid culture supplemented with zeocin and uracil or histidine as appropriate for 2 weeks and serial dilutions were plated on selective media with uracil or histidine to isolate sub-clones. Sub-clones were then screened for homozygous PtUMPS or PtPRA-PH/CH knockout phenotypes by replica streaking on minimal L1 media and L1 media supplemented with uracil or histidine as appropriate. Streaks were grown for 5 days before visual identification of phenotypes. Sub-clones that were identified as phenotypic knockouts were resuspended in TE buffer and flash frozen at $-80^{\circ} \mathrm{C}$ followed by heating at $95^{\circ} \mathrm{C}$ to lyse cells and extract genomic DNA, then sgRNA target sites were PCR amplified. Sanger sequencing of PCR products was performed at the London Regional Genomics Facility to identify the type and length of indels generated. Stable bi-allelic PtUMPS or PtPRA-PH/CH knockout mutant lines were then grown in nonselective L1 media supplemented with uracil or histidine for 1 week to cure them of plasmids before plating to obtain single colonies. Resulting colonies were replica streaked onto nonselective and zeocin-containing media supplemented with uracil or histidine to identify colonies which had successfully been cured of the plasmid. 
A

Initial concentration $\left(x 10^{4}\right.$ cells plaque $\left.{ }^{-1}\right)$

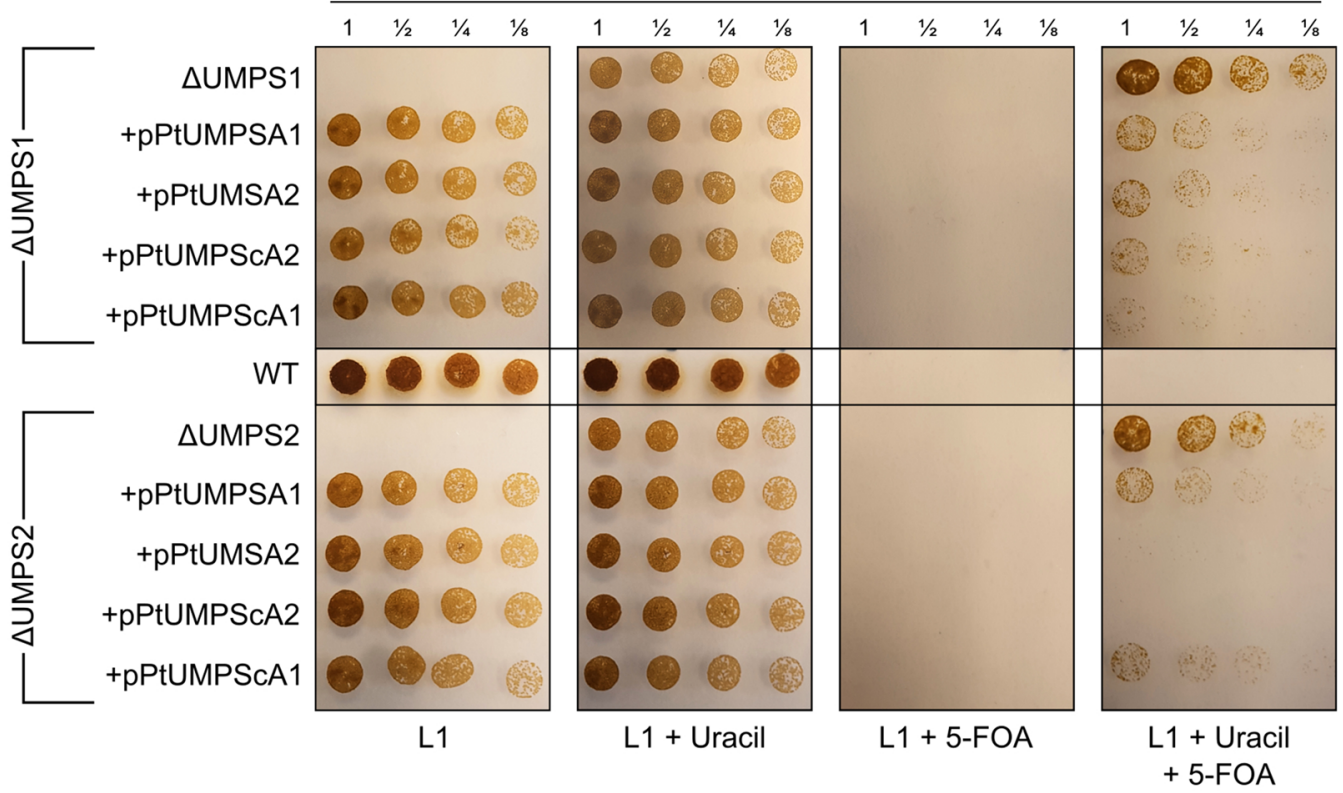

B
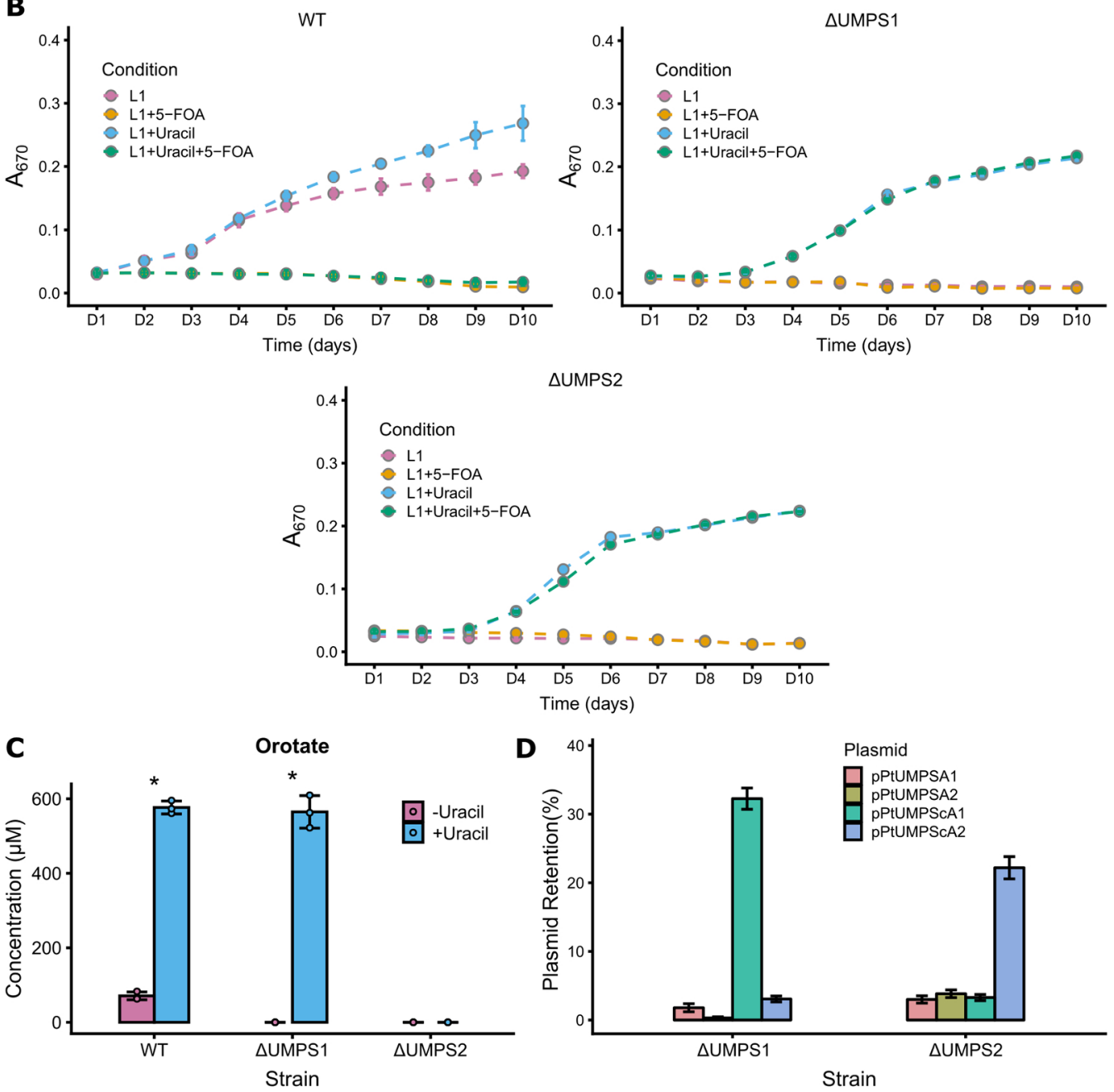
Spot plating $P$. tricornutum. Cultures of $P$. tricornutum were adjusted to $1 \times 10^{6}$ cells $\mathrm{mL}^{-1}$ and serially diluted $2 \mathrm{X}$ three times. For uracil auxotrophs, $10 \mu \mathrm{L}$ of each adjusted culture and dilutions were spot plated onto minimal L1 media and L1 media supplemented with uracil $\left(50 \mathrm{mg} \mathrm{L}^{-1}\right), 5$-FOA $\left(100 \mathrm{mg} \mathrm{L}^{-1}\right)$, or both. For histidine auxotrophs, $10 \mu \mathrm{L}$ of each adjusted culture and dilutions were spot plated onto minimal L1 media and $\mathrm{L} 1$ media supplemented with histidine $\left(200 \mathrm{mg} \mathrm{L}^{-1}\right)$. Plates were incubated at $18^{\circ} \mathrm{C}$ under cool white fluorescent lights $\left(75 \mu \mathrm{E} \mathrm{m}^{-2} \mathrm{~s}^{-1}\right)$ and a photoperiod of $16 \mathrm{~h}$ light: $8 \mathrm{~h}$ dark for 7-10 days.

Measuring $P$. tricornutum growth rates. Growth was measured in a Multiskan Go microplate spectrophotometer. Cultures of each strain (WT, $\triangle$ UMPS1, $\triangle$ UMPS1 + pPtUMPS40S, $\triangle$ UMPS1 + pPtUMPSA1, $\triangle \mathrm{UMPS} 1+$ pPtUMPSA2, $\triangle \mathrm{UMPS} 1+$ pPtUMPScA1, $\triangle \mathrm{UMPS} 1+$ pPtUMPScA2, $\triangle \mathrm{UMPS} 2+$ pPtUMPSA1, $\Delta$ UMPS2 + pPtUMPSA2, $\triangle$ UMPS2 + pPtUMPScA1, $\triangle$ UMPS2 + pPtUMPScA2) were adjusted to $5 \times 10^{5}$ cells $\mathrm{mL}^{-1}$ in L1 media with and without supplemented uracil $\left(50 \mathrm{mg} \mathrm{L}^{-1}\right), 5$-FOA $\left(100 \mathrm{mg} \mathrm{L}^{-1}\right)$, or both. Two hundred microliters of each adjusted culture was added to three wells (technical replicates) of a 96-well microplate. The 96-well microplates were incubated at $18{ }^{\circ} \mathrm{C}$ under cool white fluorescent lights $\left(75 \mu \mathrm{E} \mathrm{m}^{-2} \mathrm{~s}^{-1}\right)$ and a photoperiod of $16 \mathrm{~h}$ light:8 h dark for 10 days, and absorbance at $670 \mathrm{~nm}\left(\mathrm{~A}_{670}\right)$ was measured every $24 \mathrm{~h}$. The 96-well microplates were shaken briefly to resuspend any settled cells prior to absorbance measurements. Note that the $\mathrm{A}_{670}$ was not adjusted for path length and light scattering from the microplate lid and is therefore not directly comparable to optical density readings measured in a standard cuvette.

$P$. tricornutum metabolite extraction. Cultures of $P$. tricornutum (Wild-type, $\Delta$ UMPS1, and $\triangle \mathrm{UMPS} 2$ ) were grown with and without uracil supplementation and harvested during exponential phase as follows (Note: The $\triangle$ UMPS1 and $\triangle$ UMPS2 cultures were first grown with uracil supplementation, then switched to minimal L1 media for 1 week prior to harvesting). Cultures $\left(\sim 1 \times 10^{9}\right.$ cells) were pelleted by centrifugation at $4000 \times g$ for 10 $\mathrm{min}$ and washed by resuspending in fresh L1 media. Cells were pelleted again, resuspended in a small volume $(\sim$ $5 \mathrm{~mL}$ ) of $\mathrm{L} 1 \mathrm{media}$, and transferred to a clean $10 \mathrm{~mL}$ syringe (without needle) with the exit plugged by parafilm. The syringe was placed, tip-down, into a clean $50 \mathrm{~mL}$ falcon tube and the cells were pelleted as above. The supernatant was removed and the pellet was slowly ejected from the syringe into a pre-chilled mortar containing liquid nitrogen. The frozen cells were ground to a fine powder and then transferred to a clean pre-weighed $1.5 \mathrm{~mL}$ Eppendorf tube, suspended half way in liquid nitrogen. Being careful to keep samples frozen, $50 \mathrm{mg}$ of frozen ground powder was weighed out into a new clean $1.5 \mathrm{~mL}$ Eppendorf tube, pre-cooled in liquid nitrogen, and 250 $\mu \mathrm{L}$ of cold extraction buffer with internal standard (IS) (80\% methanol in MilliQ water, $125 \mu \mathrm{M}^{15} \mathrm{~N}_{2}-$ uracil) was added. The IS was added to the samples to compensate for losses that might occur during preparation of the samples and loss of sensitivity attributable to quenching of the signal by coeluting compounds. Samples were then homogenized by vigorous vortexing for $30 \mathrm{~s}$ in 10 second intervals, between which samples are kept on ice for $\sim 30 \mathrm{~s}$. Homogenized samples were then spun down at $4^{\circ} \mathrm{C}$ for $10 \mathrm{~min}$ at $20,000 \times g$. The supernatant was transferred to a new clean $1.5 \mathrm{~mL}$ Eppendorf tube and spun down at $20,000 \times \mathrm{g}$ for $5 \mathrm{~min}$ at $4{ }^{\circ} \mathrm{C}$. The supernatant was again transferred to a new clean $1.5 \mathrm{~mL}$ Eppendorf tube and kept at $4^{\circ} \mathrm{C}$ overnight prior to LC-MS analysis.

Chromatographic separation and mass spectrometry. Metabolites were separated at $45^{\circ} \mathrm{C}$ on a Waters Acquity HSS T3 column $[2.1 \times 100 \mathrm{~mm}, 1.8 \mu \mathrm{m}]$ in a Waters ACQUITY UPLC I-Class system (Waters, Milford, MA). Solvent A consisted of water and solvent B consisted of methanol, both containing $0.1 \%$ formic acid. Elution was performed by use of a linear gradient, at a flow rate of $0.3 \mathrm{~mL} / \mathrm{min}$, as follows: $0-2 \mathrm{~min}, 100 \%$ solvent A to $90 \%$ solvent B; $2.01 \mathrm{~min}, 100 \%$ solvent A to recondition the column. A Waters Xevo G2-S quadrupole time of flight mass spectrometer was operated in negative electrospray ionization (ESI) in resolution mode. The capillary voltage was set to $1.0 \mathrm{kV}$, the source temperature was $150{ }^{\circ} \mathrm{C}$, desolvation temperature was $600{ }^{\circ} \mathrm{C}$, the cone gas was $50 \mathrm{~L} / \mathrm{h}$ and the desolvation gas was $1000 \mathrm{~L} / \mathrm{h}$. Leucine enkephalin was infused as the lock mass with a scan time of 0.3 seconds every $10 \mathrm{~s}$, and three scans were averaged. Linearity and detection limits for each compound were established by injection of calibration mixtures with different concentrations $(0,1,2,4,8$, $16,31.25,62.5,125,250$, and $500 \mu \mathrm{mol} / \mathrm{L})$. Stable-isotope-labeled uracil $\left({ }^{15} \mathrm{~N}^{2}-\right.$ uracil $)$ was used as the IS. The concentration of each analyte was determined by use of the slope and intercept of the calibration curve that was obtained from a least-squares regression for the analyte/IS peak-area ratio vs the concentration of the analyte in the calibration mixture.

P. tricornutum DNA extraction and targeted long-read sequencing. Plasmids pPtGE34 or pPtGE35, containing sgRNAs targeting the PtUMPS, PtUrease, or PtI3GPS-PRAI gene were conjugated from $E$. coli to $P$. tricornutum and exconjugants were selected on zeocin-containing media, supplemented with uracil or tryptophan $\left(100 \mathrm{mg} \mathrm{L}^{-1}\right)$ as appropriate. For each transformation, colonies $(\sim 1,000)$ were scraped and pooled in liquid L1 media and genomic DNA was extracted using a modified akaline lysis protocol as follows: Cells were pelleted at $4,000 \times \mathrm{g}$ for $5 \mathrm{~min}$, and resuspended in $250 \mu \mathrm{L}$ resuspension buffer consisting of $235 \mu \mathrm{L}$ P1 (Qiagen), 5 $\mu \mathrm{L}$ hemicellulose $100 \mathrm{mg} \mathrm{mL}^{-1}, 5 \mu \mathrm{L}$ of lysozyme $25 \mathrm{mg} \mathrm{mL}^{-1}$, and $5 \mu \mathrm{L}$ zymolyase solution $(200 \mathrm{mg}$ zymolyase

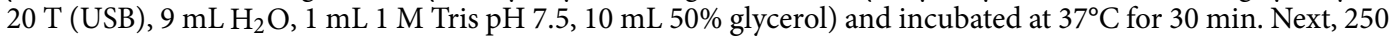
$\mu \mathrm{L}$ of lysis buffer P2 (Qiagen) was added, followed by $250 \mu \mathrm{L}$ of neutralization buffer P3 (Qiagen) and centrifugation at $16,000 \times g$ for $10 \mathrm{~min}$. The supernatant was transferred to a clean tube, $750 \mu \mathrm{L}$ isopropanol was added, and the samples centrifuged at $16,000 \times g$ for $10 \mathrm{~min}$. A $70 \% \mathrm{EtOH}$ wash was performed, centrifuged at $16,000 \times \mathrm{g}$ for $5 \mathrm{~min}$, and pellets briefly dried, resuspended in $50-100 \mu \mathrm{L}$ of TE buffer, and incubated at $37^{\circ} \mathrm{C}$ for $30-60 \mathrm{~min}$.

The sgRNA target site regions were PCR amplified from sgRNA transformant genomic DNA samples, as well as a wild-type sample, with PrimeStar GXL polymerase (Takara) using primers positioned $\sim 3 \mathrm{~kb}$ up- and downstream of the target site (Supplementary Table S6). PCR products were purified and DNA libraries were 
prepared, barcoded, and pooled using an Oxford Nanopore Ligation Sequencing Kit (SQK LSK109) and Native Barcoding Expansion 1-12 (EXP-NBD104) kit according to manufacturers protocol with the following modification-all reactions were scaled down to half the recommended volume and the end prep incubation times were extended to $15 \mathrm{~min}$ at $20^{\circ} \mathrm{C}$ and $15 \mathrm{~min}$ at $65^{\circ} \mathrm{C}$. The pooled library was then loaded on to a MinION R9.4.1 flowcell and sequenced.

Targeted long-read sequencing analysis. After sequencing on an R9.4.1 flowcell, base calling was performed using GPU Guppy with the high accuracy configuration file version 3.4.4 (https://community.nanop oretech.com). Reads in each barcode were filtered using NanoFilt ${ }^{42}$ for a minimum average read quality score of 10 and a minimum read length of 2,000, mapped using minimap $2^{43}$ and filtered for reads that map to within 100 bases of each end of the reference sequence (the unedited $6 \mathrm{~kb}$ PCR product sequence) to remove short fragments. The filtered reads were mapped using minimap2 (parameters:-ax map-ont) and outputted in sam format, then converted to bam, sorted, and indexed using samtools ${ }^{44}$. The per-base coverage depth for each barcode was calculated using Mosdepth ${ }^{45}$. All plots were created in R using the ggplot2 package ${ }^{46}$.

\section{Data availability}

The cDNA sequences of PtUMPS and PtPRA-PH/CH alleles have been deposited to GenBank under accession codes MN242208, MN242209, MN242210, and MN242211.

Received: 20 April 2020; Accepted: 27 July 2020

Published online: 17 August 2020

\section{References}

1. Scaife, M. A. \& Smith, A. G. Towards developing algal synthetic biology. Biochem. Soc. Trans. 44, 716-22 (2016).

2. Wang, B., Wang, J. \& Meldrum, D. R. Application of synthetic biology in cyanobacteria and algae. Front. Microbiol. 3, 1-15 (2012).

3. Yongmanitchai, W. \& Ward, O. P. Growth of and omega-3 fatty acid production by Phaeodacytlum tricornutum under different culture conditions. Appl. Environ. Microbiol. 57, 419-425 (1991).

4. Bozarth, A., Maier, U. G. \& Zauner, S. Diatoms in biotechnology: Modern tools and applications. Appl. Microbiol. Biotechnol. 82(2), 195-201 (2009).

5. Karas, B. J. et al. Designer diatom episomes delivered by bacterial conjugation. Nat. Commun. 6, 6925 (2015).

6. Slattery, S. S. et al. an expanded plasmid-based genetic toolbox enables Cas 9 genome editing and stable maintenance of synthetic pathways in Phaeodacytlum tricornutum. ACS Synth. Biol. 7, 328-338 (2018).

7. Serif, M. et al. One-step generation of multiple gene knock-outs in the diatom Phaeodacytlum tricornutum by dna-free genome editing. Nat. Commun. 9, 3924 (2018).

8. Stukenberg, D. D., Zauner, S. S., Dell Aquila, G. \& Maier, U. G. Optimizing crispr/cas9 for the diatom Phaeodacytlum tricornutum. Front. Plant Sci. 9, 740 (2018).

9. Sharma, A. K., Nymark, M., Sparstad, T., Bones, A. M. \& Winge, P. Transgene-free genome editing in marine algae by bacterial conjugation-comparison with biolistic crispr/cas9 transformation. Sci. Rep. 8, 14401 (2018).

10. Moosburner, M. A. et al. Multiplexed knockouts in the model diatom phaeodactylum by episomal delivery of a selectable cas9. Front. Microbiol.https://doi.org/10.3389/fmicb.2020.00005 (2020).

11. Hopes, A., Nekrasov, V., Kamoun, S. \& Mock, T. Editing of the urease gene by crispr-cas in the diatom Thalassiosira pseudonana. Plant Methods 12, 49 (2016).

12. Gatignol, A., Durand, H. \& Tiraby, G. Bleomycin resistance conferred by a drug-binding protein. FEBS Lett. 230, 171-5 (1988).

13. Zaslavskaia, L. A., Casey Lippmeier, J., Kroth, P. G., Grossman, A. R. \& Apt, K. E. Transformation of the diatom Phaeodacytlum tricornutum (Bacillariophyceae) with a variety of selectable marker and reporter genes. J. Phycol. 36, 379-386 (2000).

14. Krügel, H., Fiedler, G., Smith, C. \& Baumberg, S. Sequence and transcriptional analysis of the nourseothricin acetyltransferaseencoding gene nat1 from Streptomyces noursei. Gene 127, 127-131 (1993).

15. Buck, J. M., Bártulos, C. R., Gruber, A. \& Kroth, P. G. Blasticidin-s deaminase, a new selection marker for genetic transformation of the diatom Phaeodacytlum tricornutum. PeerJ 6, e5884 (2018).

16. Chassy, B. M. Food safety evaluation of crops produced through biotechnology. J. Am. Coll. Nutr. 21, 166S-173S (2002).

17. Romero, J., Feijoó, C. G. \& Navarrete, P. Antibiotics in aquaculture-use, abuse and alternatives. Health Environ. Aquacult. 159, (2012).

18. Peterbauer, C., Maischberger, T. \& Haltrich, D. Food-grade gene expression in lactic acid bacteria. Biotechnol. J. 6, 1147-1161 (2011).

19. Pronk, J. T. Auxotrophic yeast strains in fundamental and applied research. Appl. Environ. Microbiol. 68, 2095-2100 (2002).

20. Newman, E., Miller, B., Colebrook, L. \& Walker, C. A mutation in Escherichia coli k-12 results in a requirement for thiamine and a decrease in l-serine deaminase activity. J. Bacteriol. 161, 272-276 (1985).

21. Goldstein, A., Goldstein, D. B., Brown, B. J. \& Chou, S.-C. Amino acid starvation in an Escherichia coli auxotroph: I. Effects on protein and nucleic acid synthesis and on cell division. Biochim. et Biophys. Acta 36, 163-172 (1959).

22. Sakaguchi, T., Nakajima, K. \& Matsuda, Y. Identification of the UMP synthase gene by establishment of uracil auxotrophic mutants and the phenotypic complementation system in the marine diatom Phaeodacytlum tricornutum. Plant Physiol. 156, 78-89 (2011).

23. Kanehisa, M. \& Goto, S. Kegg: Kyoto encyclopedia of genes and genomes. Nucleic Acids Res. 28, 27-30 (2000).

24. Kanehisa, M., Sato, Y., Furumichi, M., Morishima, K. \& Tanabe, M. New approach for understanding genome variations in kegg. Nucleic Acids Res. 47, D590-D595 (2019).

25. Chiariotti, L., Alifano, P., Carlomagno, M. S. \& Bruni, C. B. Nucleotide sequence of the escherichia coli hisd gene and of the Escherichia coli and Salmonella typhimurium hisie region. Mol. Gen.Genet. MGG 203, 382-388 (1986).

26. Ingle, R. A. Histidine Biosynthesis. Arabidopsis Book 1-9, (2011).

27. Wolfs, J. M. et al. Biasing genome-editing events toward precise length deletions with an RNA-guided TevCas9 dual nuclease. Proc. Natl. Acad. Sci. U.S.A. 113, 14988-14993 (2016).

28. Russo, M. T., Cigliano, R. A., Sanseverino, W. \& Ferrante, M. I. Assessment of genomic changes in a crispr/cas9 Phaeodacytlum tricornutum mutant through whole genome resequencing. PeerJ 6, e5507 (2018).

29. Kosicki, M., Tomberg, K. \& Bradley, A. Repair of double-strand breaks induced by crispr-cas9 leads to large deletions and complex rearrangements. Nat. Biotechnol. 36, 765-771 (2018).

30. Gorter de Vries, A. R. et al. Allele-specific genome editing using crispr-cas 9 is associated with loss of heterozygosity in diploid yeast. Nucleic Acids Res. 47, 1362-1372 (2019). 
31. Weyman, P. D. et al. Inactivation of Phaeodacytlum tricornutum urease gene using transcription activator-like effector nucleasebased targeted mutagenesis. Plant Biotechnol. J. 13, 460-470 (2015).

32. Daboussi, F. et al. Genome engineering empowers the diatom Phaeodacytlum tricornutum for biotechnology. Nat. Commun. $\mathbf{5}$, 1-7 (2014).

33. Yeh, C. D., Richardson, C. D. \& Corn, J. E. Advances in genome editing through control of dna repair pathways. Nat. Cell Biol. 1-11, 1468 (2019).

34. Angstenberger, M., Krischer, J., Aktaş, O. \& Bächel, C. Knock-down of a ligiv homologue enables dna integration via homologous recombination in the marine diatom Phaeodacytlum tricornutum. ACS Synth. Biol. 8, 57-69 (2018).

35. Certo, M. T. et al. Tracking genome engineering outcome at individual dna breakpoints. Nat. Methods 8, 671 (2011).

36. Serif, M., Lepetit, B., Weißert, K., Kroth, P. \& Rio Bartulos, C. A fast and reliable strategy to generate TALEN-mediated gene knockouts in the diatom Phaeodacytlum tricornutum. Algal Res. 23, 186-195 (2017).

37. Mali, P. et al. Cas 9 transcriptional activators for target specificity screening and paired nickases for cooperative genome engineering. Nat. Biotechnol. 31, 833 (2013).

38. Karas, B. J. et al. Designer diatom episomes delivered by bacterial conjugation. Nat. Commun. 6, 1-10 (2015).

39. Noskov, V. N. et al. Assembly of large, high G+C bacterial DNA fragments in yeast. ACS Synth. Biol. 1, 267-273 (2012).

40. Gibson, D. G. Synthesis of DNA fragments in yeast by one-step assembly of overlapping oligonucleotides. Nucleic Acids Res. 37, 6984-6990 (2009).

41. Wang, H., Slattery, S., Karas, B. \& Edgell, D. Delivery of the cas9 or tevcas9 system into Phaeodacytlum tricornutum via conjugation of plasmids from a bacterial donor. Bio-Protocol 8, e2974 (2018).

42. Visualizing and processing long-read sequencing data. De Coster, W., D Hert, S., Schultz, D. T., Cruts, M. \& Van Broeckhoven, C. Nanopack. Visualizing and processing long-read sequencing data. Bioinformatics 34, 2666-2669 (2018).

43. Li, H. Minimap2: Pairwise alignment for nucleotide sequences. Bioinformatics 34, 3094-3100 (2018).

44. Li, H. et al. The sequence alignment/map format and samtools. Bioinformatics 25, 2078-2079 (2009).

45. Pedersen, B. S. \& Quinlan, A. R. Mosdepth: Quick coverage calculation for genomes and exomes. Bioinformatics 34, 867-868 (2018).

46. Wickham, H. ggplot2: Elegant Graphics for Data Analysis (Springer, New York, 2016).

\section{Acknowledgements}

This work was supported by Natural Sciences and Engineering Research Council of Canada (NSERC) Discovery Grants [RPGIN-2015-04800 to D.R.E. and RGPIN-2018-06172 to B.J.K.]. We thank Greg Gloor for advice on Nanopore sequencing and data analysis.

\section{Author contributions}

S.S.S., D.J.G., B.L.U., B.J.K. and D.R.E. conceived the experiments, S.S.S., H.W., C.K., D.J.G. and B.L.U. performed the experiments, S.S.S, D.J.G., B.J.K. and D.R.E. analysed the results, S.S.S and D.R.E. wrote the manuscript. All authors reviewed the manuscript.

\section{Competing interests}

The authors declare no competing interests.

\section{Additional information}

Supplementary information is available for this paper at https://doi.org/10.1038/s41598-020-70769-6.

Correspondence and requests for materials should be addressed to D.R.E.

Reprints and permissions information is available at www.nature.com/reprints.

Publisher's note Springer Nature remains neutral with regard to jurisdictional claims in published maps and institutional affiliations.

Open Access This article is licensed under a Creative Commons Attribution 4.0 International License, which permits use, sharing, adaptation, distribution and reproduction in any medium or format, as long as you give appropriate credit to the original author(s) and the source, provide a link to the Creative Commons licence, and indicate if changes were made. The images or other third party material in this article are included in the article's Creative Commons licence, unless indicated otherwise in a credit line to the material. If material is not included in the article's Creative Commons licence and your intended use is not permitted by statutory regulation or exceeds the permitted use, you will need to obtain permission directly from the copyright holder. To view a copy of this licence, visit http://creativecommons.org/licenses/by/4.0/.

(C) The Author(s) 2020 